\title{
An Interpretable Deep Learning Model for EEG Signals
}

\author{
Amirhessam Tahmassebi ${ }^{1}$, Anke Meyer-Baese $^{1}$, and Amir Gandomi ${ }^{2}$ \\ ${ }^{1}$ Florida State University \\ ${ }^{2}$ University of Technology Sydney
}

June 15, 2020

\begin{abstract}
Cutting-edge methods in artificial intelligence (AI) have the ability to significantly improve outcomes. However, the struggle to interpret these black box models presents a serious problem to the industry. When selecting a model, the decision to sacrifice accuracy for interpretability must be made. In this paper, we consider a case study on eye state detection using electroencephalogram (EEG) signals to investigate how a deep neural network (DNN) model makes a prediction, and how that prediction can be interpreted.
\end{abstract}

\section{Hosted file}

AI_Letter_Wiley_EEG_DNN_SHAP.pdf available at https://authorea.com/users/318151/articles/ 459730-an-interpretable-deep-learning-model-for-eeg-signals 